\title{
BMJ Open Functional, oncological outcomes and safety of nephron-sparing surgery versus radical nephrectomy in patients with localised renal cell carcinoma with high anatomical complexity: a retrospective cohort study with propensity score matching method
}

\author{
Xuanyu Zhang, ${ }^{1}$ Zhonghua Su, ${ }^{2}$ Peng Lv, ${ }^{1}$ Zeqi Liu, ${ }^{1}$ Song Bai (D) ${ }^{1}$
}

To cite: Zhang X, Su Z, Lv P, et al. Functional, oncological outcomes and safety of nephron-sparing surgery versus radical nephrectomy in patients with localised renal cell carcinoma with high anatomical complexity: a retrospective cohort study with propensity score matching method. BMJ Open 2021;11:e051622. doi:10.1136/ bmjopen-2021-051622

\section{- Prepublication history and} additional supplemental material for this paper are available online. To view these files, please visit the journal online (http://dx.doi.org/10.1136/ bmjopen-2021-051622).

Received 24 March 2021 Accepted 30 November 2021

Check for updates

(c) Author(s) (or their employer(s)) 2021. Re-use permitted under CC BY-NC. No commercial re-use. See rights and permissions. Published by BMJ.

For numbered affiliations see end of article.

Correspondence to

Dr Song Bai;

baisongcmu81@163.com

\section{ABSTRACT}

Background Nephron-sparing surgery (NSS) is widely applied for small renal masses. However, the indication of NSS in patients with localised renal cell carcinoma (RCC) with high anatomical complexity is controversial. Thus, we compare functional and oncological outcomes, and safety of NSS versus radical nephrectomy (RN) in patients with localised RCC with high anatomical complexity Radius, Exophytic/endophytic, Nearness, Anterior/posterior, Location. (R.E.N.A.L.) score $\geq 10$.

Methods We evaluated 575 patients with localised RCC that underwent NSS or RN at our centre between January 2013 and December 2018. Demographic characteristics, comorbidities, tumour data, surgery procedure, perioperative data and survival data were recorded. After propensity score matching, the variables were compared by binary paired logistic regression. The change in estimated glomerular filtration rate (eGFR) was compared with covariance analysis adjusted for baseline value. Recurrence and survival were calculated using Kaplan-Meier curves with log-rank tests. $\mathrm{P}<0.05$ was considered to be statistically significant.

Results In the matched group, NSS showed lower eGFR loss compared with the RN group (17.81 mL/ $\mathrm{min} / 1.73 \mathrm{~m}^{2}$ vs $\left.28.28 \mathrm{~mL} / \mathrm{min} / 1.73 \mathrm{~m}^{2}, \mathrm{p}<0.001\right)$. Moreover, the recurrence-free survival $(p=0.002)$, cardiocerebrovascular disease-specific survival $(p=0.015)$ and overall survival $(p=0.017)$ of patients with NSS were better than those that underwent RN. Cancer-specific survival in both groups showed no difference $(p=0.323)$. The incidence of minor and major complications in the two groups showed no difference ( $p=0.144, p=0.108)$.

Conclusion NSS resulted in better preservation of renal function and oncological outcomes compared with RN, with acceptable complications. These findings could help improve clinical decision making for patients with localised RCC with high anatomical complexity.
Strengths and limitations of this study

The study had a large sample size and a high followup rate.

- Propensity score matching was applied to balance baseline variables to decrease the bias.

- The R.E.N.A.L. Nephrometry Score was applied to describe anatomical complexity of renal masses.

- R.E.N.A.L. score $\geq 10$ was included in this study and such high anatomical complexity was rare in previous studies.

- This study was a retrospective study.

Trial registration number ChiCTR2000040652.

\section{INTRODUCTION}

Renal cell carcinoma (RCG) is common, accounting for almost $3 \%$ of all carcinoma with a $2 \%$ annual increase in incidence worldwide. ${ }^{1}$ It occurs at a rate of 4.4 to $11.1 / 100$ 000 persons per year. The 5-year overall survival (OS) for RCC is $49 \% .{ }^{23}$ Patients with localised RCC have a 5-year disease-specific survival of $>80 \% .^{4}$ However, up to $30 \%$ of patients with localised RCC develop tumour recurrence after treatment, ${ }^{56}$ which increases societal and financial burden.

Surgical management has been the benchmark for the treatment of localised RCC. There is now a significant improvement in the survival of patients with RCC, mainly due to the earlier detection methods and more advanced surgical management. ${ }^{7}$ Nephronsparing surgery (NSS) is widely applied for small renal masses and has better preservation of renal function, which may decrease 
the risk of cardiovascular-related and metabolic-related consequences and improve the overall prognosis compared with radical nephrectomy (RN). ${ }^{89}$ However, there is an ongoing debate over whether NSS can be applied to large renal masses with high anatomic complexity. Several multicentre studies have shown the survival benefit of NSS compared with RN in patients with RCC with tumour stage $\geq \mathrm{T} 2 .{ }^{10}{ }^{11}$ In contrast, several studies have shown equivalent survival outcomes between NSS and RN in patients with tumour size $>7 \mathrm{~cm} .{ }^{12}{ }^{13} \mathrm{In}$ addition, studies have shown that NSS might have a higher rate of complications compared with RN perioperatively. ${ }^{14}$ Therefore, only NSS is recommended for T1 renal tumours by the European Association of Urology guidelines. ${ }^{1516}$

Although a more complex surgical procedure is required, with potentially a higher rate of complications, patients with highly anatomically complex RCC that undergo NSS show better preservation of renal function compared with RN. Several studies have demonstrated that patients with chronic kidney disease (CKD) have increased risk of developing cardiovascular events, ${ }^{917}$ and that CKD was common in patients with RCC. ${ }^{18}$ Thus, the investigation of postoperative renal function change is warranted. According to previous research, studies that compared NSS and RN in highly anatomically complex RCC were sparing and often biased. Notably, most current studies have investigated patients with RCC that were only classified by tumour size, which cannot comprehensively describe the anatomical complexity. Besides, few studies have paid attention to postoperative renal function changes and cardiovascular sequelae that may play an important role in the OS.

The R.E.N.A.L. Nephrometry Score is a common scoring system to describe anatomical complexity of renal masses, in which the complexity of renal tumour scoring includes: the radius (tumour size), exophytic/ endophytic character, nearness of the tumour to the deepest portion of the collecting system or sinus, anterior/posterior descriptor and location relative to the polar line. It is stratified into three categories: low (4-6), moderate (7-9) and high. ${ }^{10-12}{ }^{19}$ We aim to compare functional, oncological outcomes and safety of NSS versus $\mathrm{RN}$ in patients with localised RCG with high anatomical complexity (R.E.N.A.L score $\geq 10$ ) using propensity score matching (PSM).

\section{METHODS}

\section{Patients}

A total of 1128 patients underwent nephrectomy in our centre between 1 January 2013 and 31 December 2018. Five hundred fifty-three patients were excluded due to the low R.E.N.A.L. score, loss of follow-up, data loss, solitary kidney, multiple tumours or bilateral tumour. In total, 575 patients were included in the final cohort (see details in figure 1).

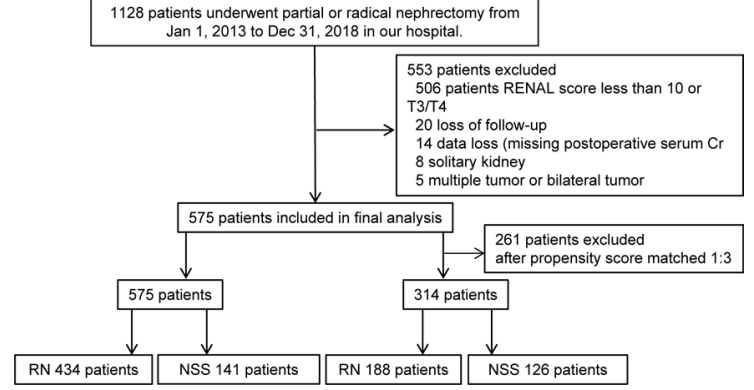

Figure 1 Flow chart of this study. Cr, creatinine; NSS, nephron-sparing surgery; $\mathrm{RN}$, radical nephrectomy.

\section{Patient and public involvement}

This study was done without involving patients or members of the public in the design, conduct, reporting and disseminating plans of the research.

\section{Inclusion and exclusion criteria}

The inclusion criteria were as follows: patients diagnosed with localised RCC by pathology (tumour stage was $\mathrm{T} 1-\mathrm{T} 2 \mathrm{~N} 0 \mathrm{M} 0{ }^{20}$; pathological type diagnosed by pathology, which includes clear-cell RCC, papillary RCC and chromophobe RCG; R.E.N.A.L. score of patients $>9$ and patients were surgically treated by NSS or RN. Patients with multiple tumours or bilateral tumours were excluded.

\section{Surgery procedure}

NSS and RN were performed using standard open or laparoscopic surgical techniques as previously reported. ${ }^{19} 20$ Open NSS was via the extraperitoneal approach with cold ischaemia, and laparoscopic NSS was via the transperitoneal approach with warm ischaemia. Finally, RN was via laparoscopy with the transperitoneal approach. All the interventions have been done by the experienced surgeon.

\section{Characteristics, outcome and follow-up}

Patient demographics were mean age, gender and body mass index (BMI). Comorbidities were hypertension, diabetes mellitus, coronary heart disease and the American Society of Anesthesiologists (ASA) scores. Tumour data were tumour side, tumour stage and pathological type. Patients with RCG were diagnosed by pathology after surgery. Intraoperative data were duration of operative time, estimated blood loss, blood transfusion rate, whether conversion to open and whether conversion to RN. Clavien-Dindo score was used to estimate the complications after surgery. ${ }^{21}$ Postoperative data were minor complications (Clavien 1-2), major complications (Clavien 3-4), urine fistula, haemorrhage need selective renal artery embolisation (SAE), perioperative mortality (Clavien $5)$, duration of postoperative hospital stay, bowel recovery (days) and time to drain removal. Prognostic data were recurrence-free survival (RFS) rate, OS rate, cardiocerebrovascular diseases-specific survival (CVDSS) rate and cancer-specific survival (CSS) at 
5 years preoperative and postoperative estimated glomerular filtration rate (eGFR). eGFR (preoperative and postoperative) were used to estimate the patients' renal function. The eGFR was based on four-variable dietary modifications in the renal disease equation. ${ }^{22}$

Follow-up protocol was every 3 months in the first 2 years, 6 months in the third year and then once a year. Follow-up examinations included physical examination, chest and abdominal CT, biochemical analysis and bone emission CT scan as necessary.

\section{Statistical analysis}

The data were collected and analysed by SPSS V.25.0 for Windows (SPSS, Chicago, Illinois, USA). Continuous variables were expressed as median (IQR); categorical variables were reported as number (percentage). Before PSM, independent samples Student's t-test was used to compare mean of two continuous normally distributed variables and the Mann-Whitney $\mathrm{U}$ test was used to compare the mean of two continuous non-normally distributed variables, The $\chi^{2}$ test or Fisher's exact test was used for categorical variables.

PSM was applied to balance baseline variables to decrease the deviation. Multivariate logistic regression analysis was used to determine propensity scores for each patient based on all baseline variables: demographic characteristics (mean age, gender and BMI), comorbidities (hypertension, diabetes mellitus, coronary heart disease and ASA), tumour data (tumour side, tumour stage and pathological type) and surgical procedure (laparoscopy vs open). The NSS and RN groups were matched 1:3 using a calliper width of 0.1 for the propensity score through the nearest neighbour matching (see details in figure 2). After PSM, the categorical variables were compared by binary conditional logistic regression. Paired samples Student's t-test was used to compare mean of two continuous normally distributed variables and the Wilcoxon test was used to compare the mean of two continuous non-normally distributed variables. Recurrence and survival were calculated using Kaplan-Meier curves with log-rank tests. The eGFR was compared with
A

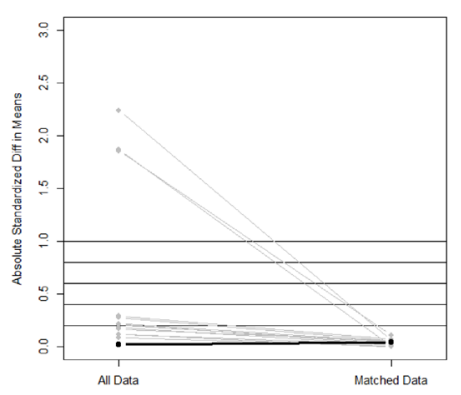

B

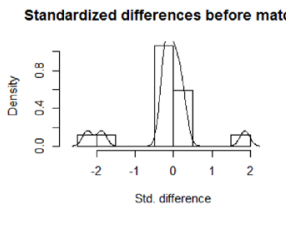

C

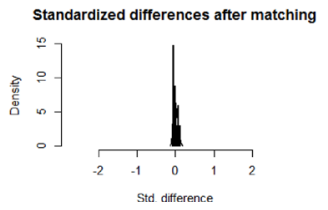

Figure 2 Plot of propensity score matching (PSM). (A) Dot plot of standardised mean differences. (B) Histogram of standardised mean differences before PSM. (C) Histogram of standardised mean differences after PSM. covariance analysis adjusted for baseline value. Twotailed $\mathrm{p}$ values $<0.05$ were considered statistically significant.

\section{Results}

In total, 575 patients included in the cohort were divided into two groups: 434 patients in the $\mathrm{RN}$ and 141 patients in the NSS. The median follow-up period of $\mathrm{RN}$ and NSS was 37.0 and 26.0 months, respectively. A balance of baseline variables between the two groups after PSM was achieved. In the matched groups, 434 patients that underwent RN and 141 patients that underwent NSS were matched with 188 patients that underwent RN and 126 patients that underwent NSS, respectively. Demographics, comorbidity, tumour characteristics and perioperative data are detailed in table 1.

In matched cohorts, the mean follow-up time was 38.0 months and 27.0 months in the RN and NSS groups, respectively. There were four variables with statistical differences. Patients that underwent NSS had a longer duration of postoperative hospital stay $(p<0.001)$, bowel recovery $(\mathrm{p}<0.001)$ and time to drainage removal $(\mathrm{p}<0.001)$. Patients in the NSS group had lower eGFR loss (eGFR change, $\mathrm{p}<0.001$ ) compared with those in the RN group (see details in table 2 ).

Patients that underwent NSS had better RFS, CVDSS and OS at 5 years than patients that underwent RN. The RFS was $95.90 \%$ vs $77.50 \%$ ( $\mathrm{p}=0.002)$, the CVDSS was $92.30 \%$ vs $75.10 \%(\mathrm{p}=0.015)$ and the OS was $95.60 \%$ vs $80.40 \%$ $(\mathrm{p}=0.017)$ in the NSS versus RN groups at 5 years, respectively. The CSS of patients was $97.30 \%$ vs $92.40 \%$, which was not statistically different ( $\mathrm{p}=0.323)$ (see details in figure 3 ).

Besides, we also compared two paired groups using multilevel regression model for baseline variables. The results of sensitivity analysis (multilevel regression model) are consistent with those of binary conditional logistic regression (see details in online supplemental table 1). We analysed these data using inverse probability treatment weighting (IPTW) for outcome variables. The results of IPTW are consistent with those of binary conditional logistic regression (see details in online supplemental table 2).

\section{DISCUSSION}

Many retrospective studies have shown that NSS has an advantage over $\mathrm{RN}$ in small renal masses due to better preservation of renal function and similar oncological control. However, NSS requires a higher level of technical skills, and has a higher rate of complications with the increasing anatomical complexity of the renal tumour. Furthermore, the studies addressing this issue are scarce and the conclusion is inconsistent. Thus, this study aimed to compare functional, oncological outcomes and safety of NSS and RN in patients with localised RCG with high anatomic complexity (R.E.N.A.L. score $\geq 10$ ). 


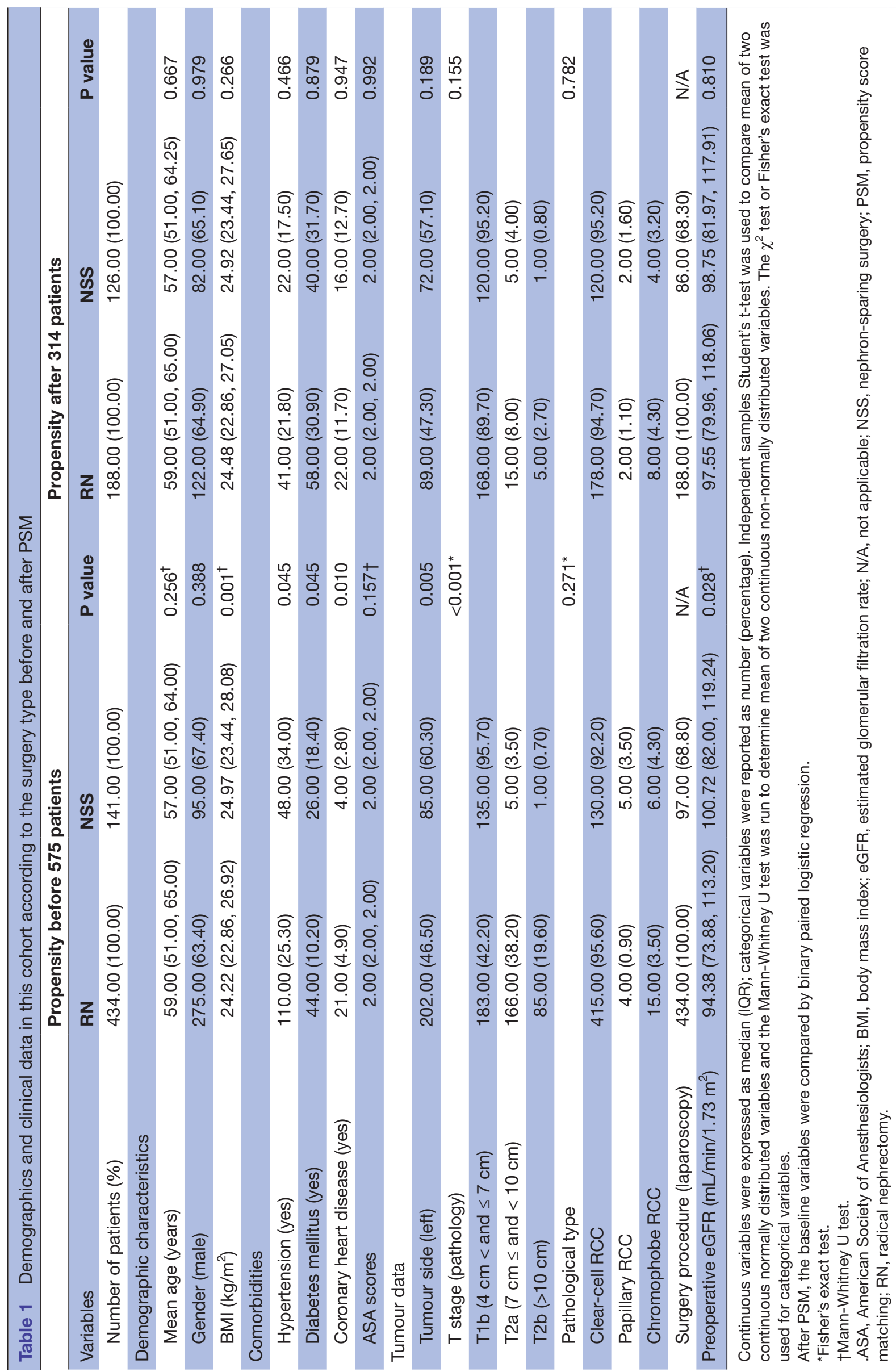

$\stackrel{0}{\mathbb{D}}$ 
A

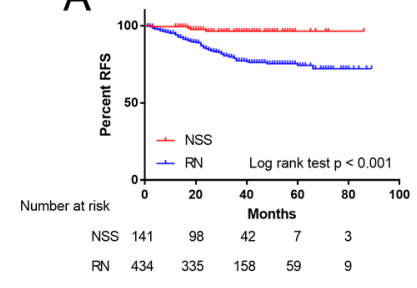

E

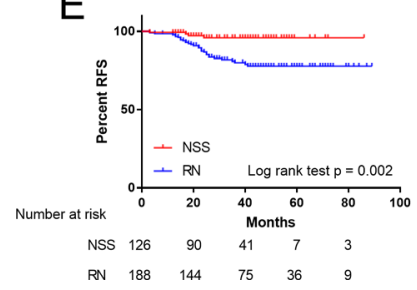

B

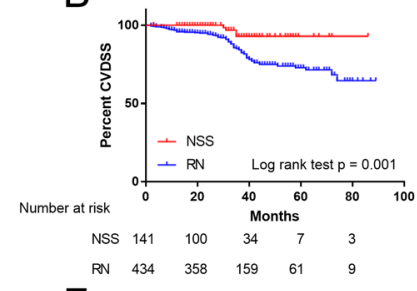

F

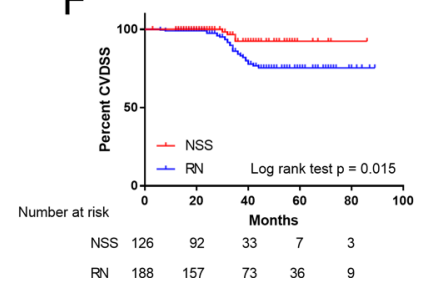

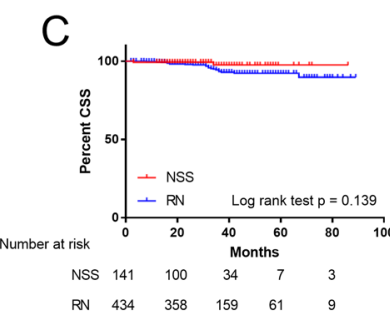

G

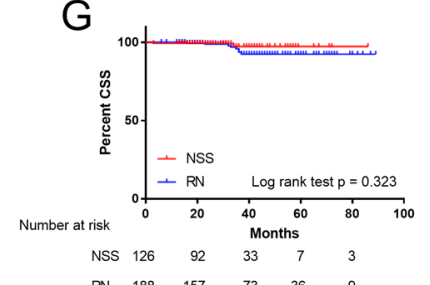

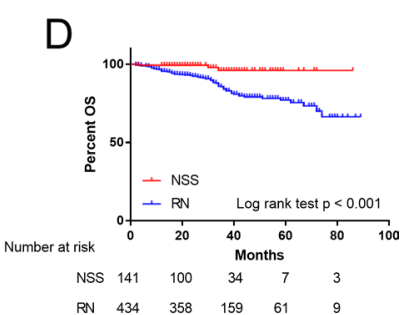

$\mathrm{H}$

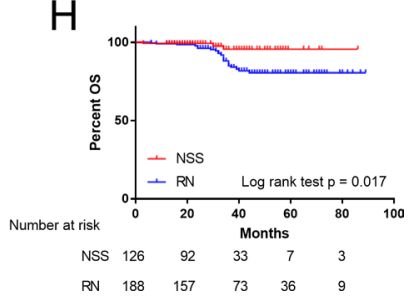

Figure 3 Survival curve stratified by surgery method before and after PSM. (A) RFS curve before PSM. (B) CVDSS curve before PSM. (C) CSS curve before PSM. (D) OS curve before PSM. (E) RFS curve after PSM. (F) CVDSS curve after PSM. (G) CSS curve after PSM. (H) OS curve after PSM. CSS, cancer-specific survival; CVDSS, cardiocerebrovascular diseasespecific survival; NSS, nephron-sparing surgery; OS, overall survival; PSM, propensity score matching; RFS, recurrence-free survival; RN, radical nephrectomy.

Ultimately, in our study, patients in the NSS group had a better preservation of renal function, higher postoperative eGFR $(p<0.001)$ and lower eGFR decrease $(p<0.001)$. In line with this study, Deng et $a l^{23}$ retrospectively studied 148 patients with RCC with a R.E.N.A.L. score $\geq 7$ and showed the same conclusion; that NSS had a better protection of renal function compared with RN. This finding may be due to there being more functioning nephrons preserved in NSS compared with RN. On the contrary, in a subgroup analysis, Kopp et $a l^{14}$ suggested NSS was lacking in renal functional benefit compared with $\mathrm{RN}$ in patients with a R.E.N.A.L. score $>10$. The possible reason for this is that the high anatomical complexity renal tumours had prolonged surgery duration and ischaemia time, which may have caused damage to the remaining renal parenchyma.

Studies have shown that if preservation of renal function is poor, it has a close association with higher occurrence of cardiovascular incidents, even with poor OS. ${ }^{24}$ Weight $e t a l^{25}$ suggested the risk of cardiac death increased by $25 \%$, due to the loss of renal function by RN. In this study, NSS had better CVDSS $(\mathrm{p}=0.015)$ and OS $(\mathrm{p}=0.017)$ compared with $\mathrm{RN}$, which is mainly due to better preservation of renal function after NSS. Consistent with this study, Deng $e t a t^{4}$ also showed that patients that underwent NSS had a longer OS than those that underwent RN. Besides, Umberto et al found that RN was associated with a higher developing postoperative hypertention risk (HR 2.89; $\mathrm{p}=0.006$ ) than NSS in patients without a medical history of cardiopathy based on a prospective cohort study. ${ }^{26}$

In this cohort, NSS had better PFS $(p=0.002)$, while there was no difference in CSS compared with RN. However, in a study comprising 123 patients with a tumour size $>7 \mathrm{~cm}$, Janssen et $a l^{27}$ showed that NSS had longer OS and CSS compared with RN. On the contrary, Jeldres $e t a l^{28}$ studied 29 patients with RCC with a tumour size $>7 \mathrm{~cm}$ in a matched analysis and showed NSS was associated with a higher rate of cancer-specific mortality.
These discrepancies were mainly due to different inclusion criteria, and here, our study only focused on patients with high anatomical complexity (R.E.N.A.L. score $\geq 10$ ).

There was no difference in either intraoperative characteristics or minor and major complications between RN and NSS in this study. However, NSS showed longer duration of postoperative hospital stay, slower bowel recovery and longer time to drainage removal. In the study by Kopp et al, ${ }^{29}$ NSS had higher risk of high-grade complications compared with RN. However, in a more recent study conducted by Deng et $a l,{ }^{23} \mathrm{NSS}$ had a higher risk of low-grade complications compared with RN, whereas there was no difference in high-grade complications between the two groups. This discrepancy may be due to the improvement in surgical technique and equipment.

The duration of postoperative hospital stay in NSS group was significantly shorter than $\mathrm{RN}$ group in this study. The main reason for this might be that there was higher rate of postoperative complication in NSS group than in RN group, such as urine fitula, haemorrhage need SAE and so on, which need more duration of hospital stay.

There were several limitations in this study. First, it was a single-centre retrospective study, which carries selection bias and historical bias. In addition, it fails to adjust for unknown confounders, even though PSM method was used. These limitations can lead to incorrect results and spurious associations. Second, robotassisted nephrectomy was excluded in this study. Although previous studies have suggested that robotassisted nephrectomy has an advantage in dealing with highly anatomically complex renal masses, ${ }^{30}$ robot-assisted nephrectomy requires higher surgical skill and a longer period of study, and may not be costeffective. This may mean robot-assisted nephrectomy will not be rapidly popularised worldwide in the short term, especially in low-income and middle-income 
countries. Third, there may have been some bias in changes of renal function between preoperative and postoperative data, which were evaluated using eGFR rather than renal scintigraphy. Fourth, the follow-up peroid of this study was short. A larger prospective randomised controlled trial in multicentre is required to verify the accuracy of the conclusion drawn from this study. Nevertheless, this study is the first to compare functional, oncological outcomes and safety of NSS and RN in patients with highly anatomically complex localised RCC.

\section{CONCLUSION}

NSS resulted in better preservation of renal function and oncological outcomes compared with RN, with acceptable complications. These findings could help improve clinical decision making for patients with localised RCC with high anatomical complexity.

\section{Author affiliations}

${ }^{1}$ Department of Urology, Shengjing Hospital of China Medical University, Shenyang, China

${ }^{2}$ Department of Obstetrics and Gynecology, Shengjing Hospital of China Medical University, Shenyang, China

Contributors SB had full access to all the data in the study and takes responsibility for the integrity of the data and the accuracy of the data analysis. Study concept and design: SB. Acquisition of data, analysis and interpretation of data: XZ, ZS, PL, ZL and SB. Drafting of the manuscript and critical revision of the manuscript for important intellectual content: XZ, ZS and SB. Statistical analysis: XZ, $\mathrm{ZS}$ and SB. Obtaining funding and other (figures): $\mathrm{SB}$ is responsible for the overall content as the guarantor for this study.

Funding This study was financially supported by the 345 Talent Project of Shengjing Hospital (grant number N/A), Natural Science Foundation of Liaoning Science and Technology Department (grant number 2020-BS-093) and Natural Science Foundation of Liaoning Education Department (grant number QN2019013). These sponsors had no role in the study design; in the collection, analysis or interpretation of data; in the writing of the report or in the decision to submit the article for publication.

Competing interests None declared.

Patient consent for publication Not applicable.

Ethics approval This study involves human participants and was approved by ethical statement. Ethical approval (ethical committee no. 2020PS678K) was provided by the Institutional Research and Ethics Committee of the Shengjing Hospital Affiliated China Medical University in Shengyang, China. Participants gave informed consent to participate in the study before taking part.

Provenance and peer review Not commissioned; externally peer reviewed.

Data availability statement Data are available on reasonable request. SB had full access to all the data in the study and takes responsibility for the integrity of the data and the accuracy of the data analysis. Data are available on reasonable request (baisongcmu81@163.com).

Supplemental material This content has been supplied by the author(s). It has not been vetted by BMJ Publishing Group Limited (BMJ) and may not have been peer-reviewed. Any opinions or recommendations discussed are solely those of the author(s) and are not endorsed by BMJ. BMJ disclaims all liability and responsibility arising from any reliance placed on the content. Where the content includes any translated material, BMJ does not warrant the accuracy and reliability of the translations (including but not limited to local regulations, clinical guidelines, terminology, drug names and drug dosages), and is not responsible for any error and/or omissions arising from translation and adaptation or otherwise.

Open access This is an open access article distributed in accordance with the Creative Commons Attribution Non Commercial (CC BY-NC 4.0) license, which permits others to distribute, remix, adapt, build upon this work non-commercially, and license their derivative works on different terms, provided the original work is properly cited, appropriate credit is given, any changes made indicated, and the use is non-commercial. See: http://creativecommons.org/licenses/by-nc/4.0/.

ORCID iD

Song Bai http://orcid.org/0000-0002-6073-145X

\section{REFERENCES}

1 Ferlay J, Colombet M, Soerjomataram I, et al. Cancer incidence and mortality patterns in Europe: estimates for 40 countries and 25 major cancers in 2018. Eur J Cancer 2018;103:356-87.

2 Li P, Wong Y-N, Armstrong K, et al. Survival among patients with advanced renal cell carcinoma in the pretargeted versus targeted therapy eras. Cancer Med 2016;5:169-81.

3 Wahlgren T, Harmenberg U, Sandström P, et al. Treatment and overall survival in renal cell carcinoma: a Swedish population-based study (2000-2008). Br J Cancer 2013;108:1541-9.

4 Elmore JM, Kadesky KT, Koeneman KS, et al. Reassessment of the 1997 TNM classification system for renal cell carcinoma. Cancer 2003;98:2329-34.

5 Haas NB, Manola J, Uzzo RG, et al. Adjuvant sunitinib or sorafenib for high-risk, non-metastatic renal-cell carcinoma (ECOG-ACRIN E2805): a double-blind, placebo-controlled, randomised, phase 3 trial. Lancet 2016;387:2008-16.

6 Lam JS, Shvarts O, Leppert JT, et al. Postoperative surveillance protocol for patients with localized and locally advanced renal cell carcinoma based on a validated prognostic nomogram and risk group stratification system. J Urol 2005;174:466-72.

7 Motzer RJ, Russo P. Systemic therapy for renal cell carcinoma. J Urol 2000;163:408-17.

8 Huang WC, Levey AS, Serio AM, et al. Chronic kidney disease after nephrectomy in patients with renal cortical tumours: a retrospective cohort study. Lancet Oncol 2006;7:735-40.

9 Go AS, Chertow GM, Fan D, et al. Chronic kidney disease and the risks of death, cardiovascular events, and hospitalization. $N$ Engl J Med 2004;351:1296-305.

10 Ljungberg B, Bensalah K, Canfield S, et al. EAU guidelines on renal cell carcinoma: 2014 update. Eur Urol 2015;67:913-24.

11 Becker F, Roos FC, Janssen M, et al. Short-Term functional and oncologic outcomes of nephron-sparing surgery for renal tumours $\geq$ $7 \mathrm{~cm}$. Eur Urol 2011:59:931-7.

12 Meskawi M, Becker A, Bianchi M, et al. Partial and radical nephrectomy provide comparable long-term cancer control for $\mathrm{T} 1 \mathrm{~b}$ renal cell carcinoma. Int J Urol 2014;21:122-8.

13 Bigot P, Hétet J-F, Bernhard J-C, et al. Nephron-sparing surgery for renal tumors measuring more than $7 \mathrm{~cm}$ : morbidity, and functional and oncological outcomes. Clin Genitourin Cancer 2014;12:e19-27.

14 Kopp RP, Mehrazin R, Palazzi KL, et al. Survival outcomes after radical and partial nephrectomy for clinical T2 renal tumours categorised by R.E.N.A.L. nephrometry score. BJU Int 2014;114:708-18.

15 MacLennan S, Imamura M, Lapitan MC, et al. Systematic review of perioperative and quality-of-life outcomes following surgical management of localised renal cancer. Eur Urol 2012;62:1097-117.

16 Van Poppel H, Da Pozzo L, Albrecht W, et al. A prospective, randomised EORTC intergroup phase 3 study comparing the oncologic outcome of elective nephron-sparing surgery and radical nephrectomy for low-stage renal cell carcinoma. Eur Urol 2011;59:543-52.

17 Anavekar NS, McMurray JJV, Velazquez EJ, et al. Relation between renal dysfunction and cardiovascular outcomes after myocardial infarction. N Engl J Med 2004;351:1285-95.

18 Dey S, Hamilton Z, Noyes SL, et al. Chronic kidney disease is more common in locally advanced renal cell carcinoma. Urology 2017; 105:101-7.

19 Kutikov A, Uzzo RG. The R.E.N.A.L. nephrometry score: a comprehensive standardized system for quantitating renal tumor size, location and depth. J Urol 2009;182:844-53.

20 Sobin LH. GM, Wittekind C, eds. TNM classification of malignant tumors. 7th ed, 2009.

21 Clavien PA, Barkun J, de Oliveira ML, et al. The clavien-dindo classification of surgical complications: five-year experience. Ann Surg 2009;250:187-96.

22 Levey AS, Bosch JP, Lewis JB, et al. A more accurate method to estimate glomerular filtration rate from serum creatinine: a new prediction equation. modification of diet in renal disease Study Group. Ann Intern Med 1999;130:461-70. 
23 Deng W, Zhou Z, Zhong J, et al. Retroperitoneal laparoscopic partial versus radical nephrectomy for large $(\geq 4 \mathrm{~cm})$ and anatomically complex renal tumors: a propensity score matching study. Eur J Surg Oncol 2020;46:1360-5.

24 Deng W, Chen L, Wang Y, et al. Partial nephrectomy versus radical nephrectomy for large $(\geq 7 \mathrm{~cm})$ renal tumors: a systematic review and meta-analysis. Urol Oncol 2019;37:263-72.

25 Weight CJ, Larson BT, Fergany AF, et al. Nephrectomy induced chronic renal insufficiency is associated with increased risk of cardiovascular death and death from any cause in patients with localized cT1b renal masses. J Urol 2010;183:1317-23.

26 Capitanio U, Larcher A, Cianflone F, et al. Hypertension and cardiovascular morbidity following surgery for kidney cancer. Eur Urol Oncol 2020;3:209-15.

27 Janssen MWW, Linxweiler J, Terwey S, et al. Survival outcomes in patients with large $(\geq 7 \mathrm{~cm})$ clear cell renal cell carcinomas treated with nephron-sparing surgery versus radical nephrectomy: results of a multicenter cohort with long-term follow-up. PLoS One 2018;13:e0196427.

28 Jeldres C, Patard J-J, Capitanio U, et al. Partial versus radical nephrectomy in patients with adverse clinical or pathologic characteristics. Urology 2009;73:1300-5.

29 Kopp RP, Liss MA, Mehrazin R, et al. Analysis of renal functional outcomes after radical or partial nephrectomy for renal masses $\geq 7$ cm Using the RENAL Score. Urology 2015;86:312-20.

30 Mari A, Tellini R, Porpiglia F, et al. Perioperative and mid-term oncological and functional outcomes after partial nephrectomy for complex (Padua score $\geq 10$ ) renal tumors: a prospective multicenter observational study (the RECORD2 project). Eur Urol Focus 2021;7:1371-9. 